\title{
THE USE OF DYNAMIC ULTRASOUND B-SCAN TO DETECT RETINAL TEARS IN SPONTANEOUS VITREOUS HAEMORRHAGE
}

\author{
K. K. NISCHAL, J. N. JAMES and J. McALLISTER \\ Windsor
}

\begin{abstract}
SUMMARY
The use of dynamic ultrasound B-scan to detect retinal tears in spontaneous vitreous haemorrhage is described. A 6-month prospective study was undertaken of non-diabetic patients who presented with spontaneous vitreous haemorrhage, in whom the fundus could not be visualised and in whom a rhegmatogenous aetiology was suspected. Patients were followed up at least weekly with repeat B-scans until adequate fundus visualisation was possible. Eight patients in total were included in the study; of these, 4 were thought to have retinal tear at initial ultrasound examination and this was confirmed later when the haemorrhage cleared. A scanning protocol is suggested.
\end{abstract}

The incidence of retinal tear (with and without detachment) in spontaneous vitreous haemorrhage, combining data from four studies, is $35 \% .{ }^{1-4}$ Ultrasonography is well established as the investigation of choice in spontaneous vitreous haemorrhage obscuring the fundus. ${ }^{5-7}$ Its use in detecting large ( $^{5,8}$ and giant $\mathrm{t}^{9}$ retinal tears in such circumstances has also been documented. Reports of detection of small to moderate tears have been scarce, retrospective in nature and have also incorporated A-scan studies. ${ }^{10}$ We present a prospective study of the use of dynamic ultrasound B-scan alone in the evaluation of retinal tears in spontaneous vitreous haemorrhages.

\section{METHOD}

Patients presenting during a 6-month period between July and December 1993 with a spontaneous vitreous haemorrhage obscuring fundus details that was suspected of having a rhegmatogenous aetiology were referred for ultrasound B-scan evaluation. Known diabetics were not referred. A $10-\mathrm{MHz}$

From: Prince Charles' Eye Unit, King Edward VII Hospital, Windsor, Berks, UK.

Correspondence to: Ken K. Nischal, Birmingham and Midland Eye Hospital, Church Street, Birmingham B3 2NS, UK. dedicated ocular probe and a contact B-scan technique through the closed eyelids were used with the patient in the upright position. A soundcoupling substance was used between the probe and the patient's skin.

B-scans in the vertical and horizontal meridians were performed with the eye in various positions of gaze, allowing examination of all four retinal quadrants, while varying the position of the probe simultaneously allowed examination of these quadrants both anterior and posterior to the equator (Figs. 1, 2). This was followed by dynamic B-scan where the patient was instructed to move the eye in various directions of gaze while the probe was held stationary. On the basis of this protocol the location of any possible tear was documented in terms of retinal quadrant. If no lesion was seen the examination was repeated with the patient lying prone on an examination couch with the head hanging down over the couch edge; this was specifically done to exclude a small inferior retinal tear.

After the first examination the patient was seen at least weekly for repeat B-scan evaluation until adequate visualisation was possible, at which point appropriate treatment was given.

\section{RESULTS}

Eight patients were referred in total: 5 women and 3 men (mean age 67 years, range 52-72 years). Four were thought to have a retinal tear, 3 in the superotemporal quadrant and 1 in the superonasal quadrant (Figs. 3-5). The other 4 haemorrhages were thought to be due to a neovascular frond in a nondiabetic (Fig. 6), an intraocular tumour (choroidal melanoma), age-related macular degeneration and acute haemorrhagic posterior vitreous detachment, respectively.

All 4 tears were confirmed as such when visualisation was possible; they were small to moderate in size and also in the predicted retinal quadrant (Fig. 7). 

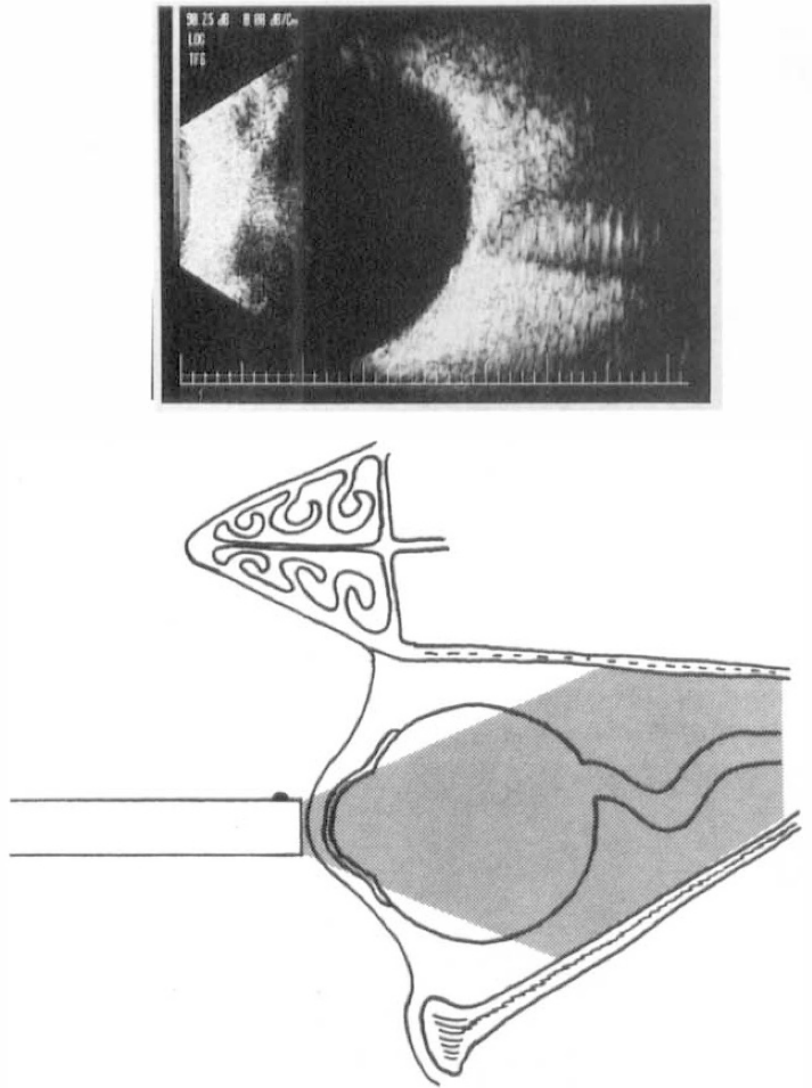

Fig. 1. Diagrammatic transverse section through the globe and optic nerve with the corresponding axial ultrasound $B$ scan shown above. If the probe is held stationary and the patient asked to adduct the eye being examined, the nasal retina is scanned; by moving the probe in the opposite direction simultaneously the extreme nasal periphery is scanned.

Prophylactic argon laser photocoagulation was successfully used to treat all 4 tears. In the other 4 cases the diagnosis was also confirmed by eventual visualisation in all but 1 ; the fundus of the patient with possible choroidal melanoma could not be adequately visualised at 6 months follow-up, though later A-scan study strongly suggested this to be the likely pathology.

No patient developed a retinal detachment on follow-up.

\section{DISCUSSION}

When data from studies on spontaneous vitreous haemorrhages ${ }^{1-4}$ are combined, the five commonest aetiologies are diabetic retinopathy $(37 \%)$, retinal tear alone $(22 \%)$, retinal detachment with retinal tear $(13 \%)$, retinal vein occlusion $(10 \%)$ and posterior vitreous detachment alone $(5 \%)$.

In our study we only used dynamic B-scan analysis. A-scan echography is most suited to tissue differentiation, ${ }^{11,12}$ but for this to be accurate the ultrasonic waves should strike the tissue being examined at right angles; the further into the periphery one tries to examine, the less easy it
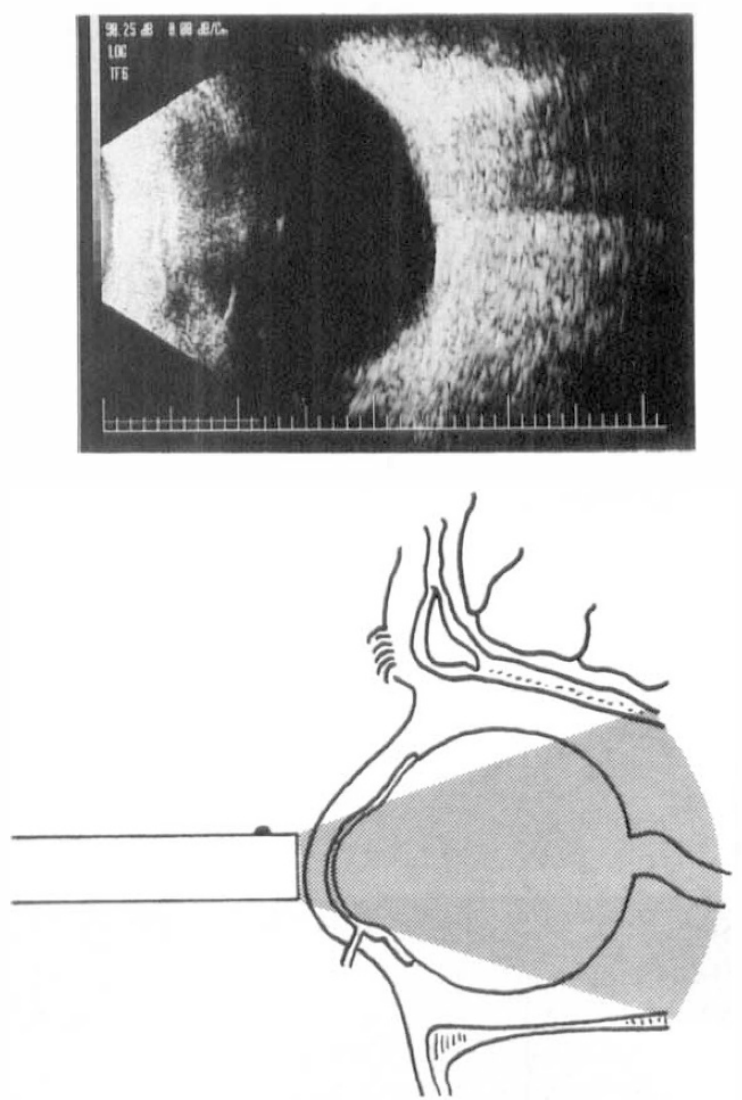

Fig. 2. Diagrammatic vertical section through the globe and optic nerve with corresponding vertical section ultrasound $B$-scan above. If the probe is held stationary and the patient looks up, the superior retina is scanned; by moving the probe down simultaneously the superior retinal periphery is scanned. Thus each quadrant can be scanned both anterior and posterior to the equator.

becomes to ensure that this condition is met. Furthermore it is recognised that using the A-scan to quantitate the reflectivity of any localised area found on B-scan is extremely difficult to accomplish and not infallible. ${ }^{11,13}$

All 4 retinal tears in our study were in the superior retina; 3 were superotemporal, which is reportedly the commonest quadrant for symptomatic retinal tears. ${ }^{14}$ The scanning protocol used is similar to one used by Jalkh et al. ${ }^{6}$ to successfully localise vitreoretinal pathology in the preoperative assessment of patients with diabetic vitreous haemorrhage.

Our study was heavily biased as diabetics were excluded and those cases thought clinically to have a rhegmatogenous aetiology (most commonly due to a history of flashing lights and/or floaters) were especially included; as a direct consequence the total number of patients is small. However, there are some important and consistent findings: all 4 cases with retinal tear on dynamic B-scan showed tears that were anterior to the equator and in the periphery and displayed a discontinuity in the retinal echo from which there extended a discrete small flap into the vitreous cavity. All 4 cases of 


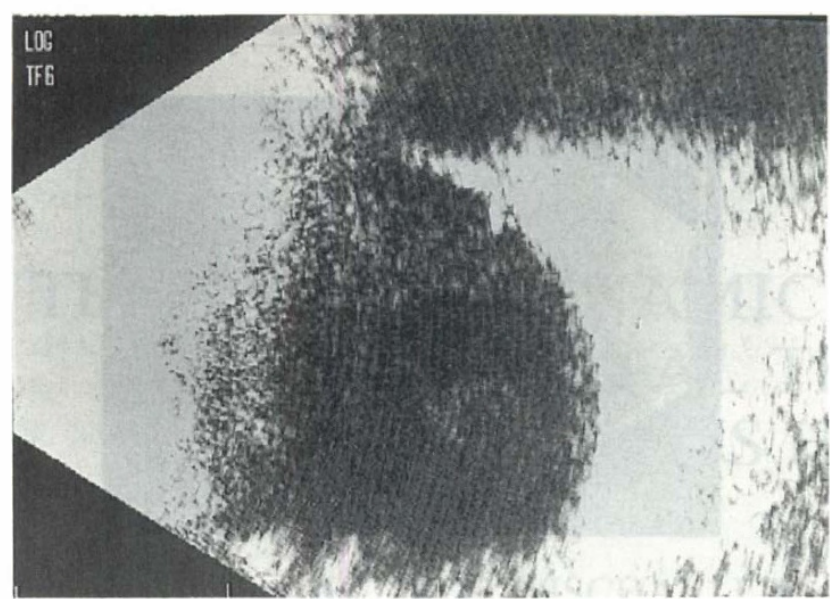

Fig. 3. Vertical section B-scan of the supero-temporal retinal quadrant showing features suggestive of a retinal tear (see text for details).

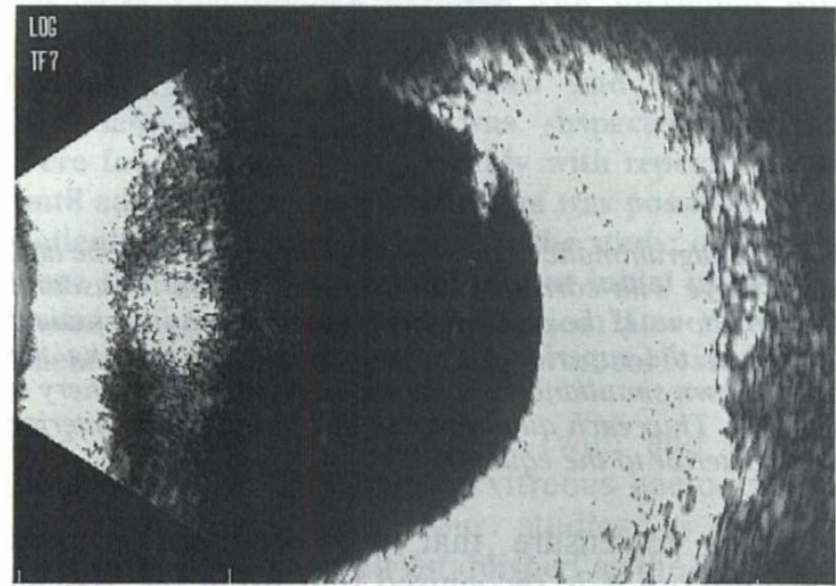

Fig. 5. Transverse B-scan of a retinal tear showing discontinuity of retinal echo. The vitreous haemorrhage is not seen since the type of enhancement used allows delineation of retinal features but not vitreal ones, and also the haemorrhage has begun to resolve.

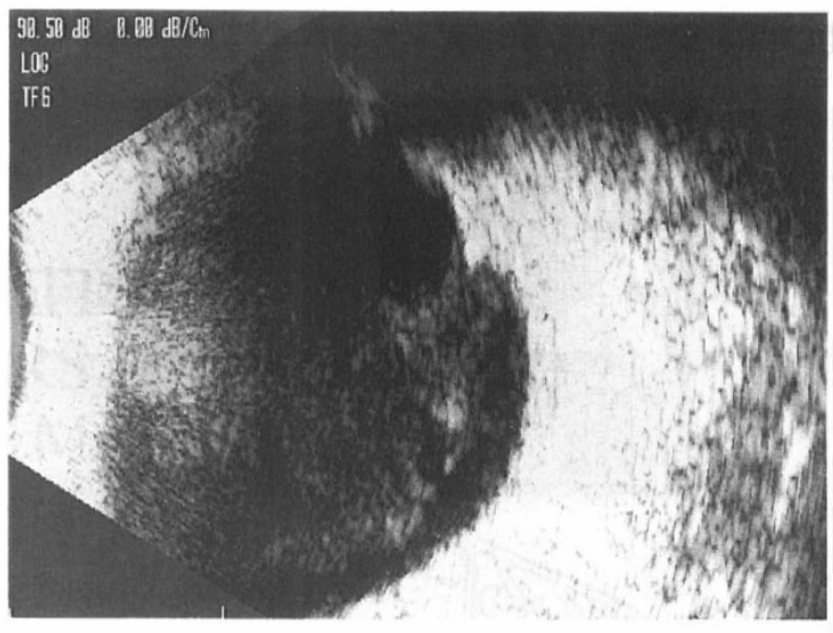

Fig. 4. Vertical section B-scan of the superior retina showing a small retinal tear with posterior vitreous face attached to the apex of the flap of the tear. Note that these are static scans and the quality of movement on dynamic scanning of this structure contributes to the examiner's diagnosis of a tear.

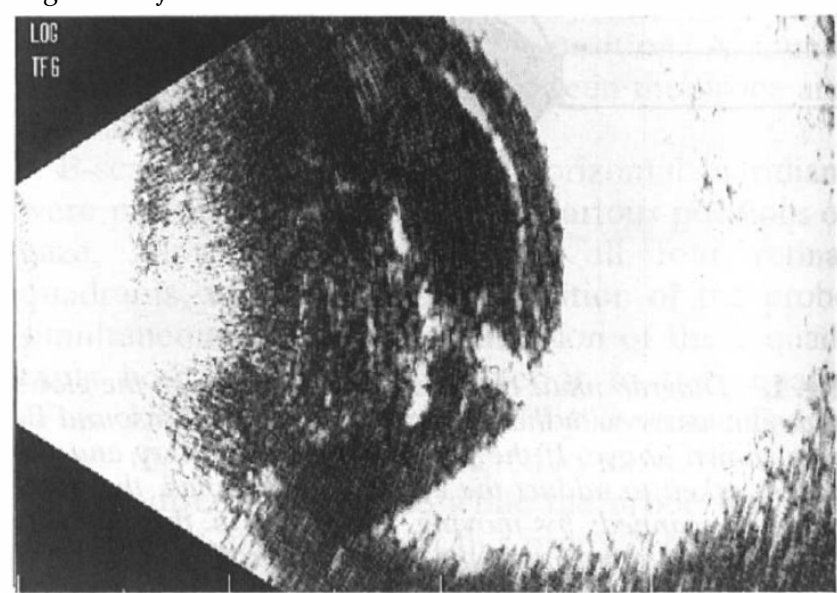

Fig. 6. Vertical section B-scan showing intragel and subhyaloid haemorrhage of the vitreous. The type of enhancement used does not normally show vitreous pathology well, but a combination of dense haemorrhage and high gain have allowed detection of the haemorrhage. There is a thick stalk-like structure extending into the vitreous cavity with acoustic enhancement at the point of attachment to the posterior vitreous face; this is a neovascular frond.

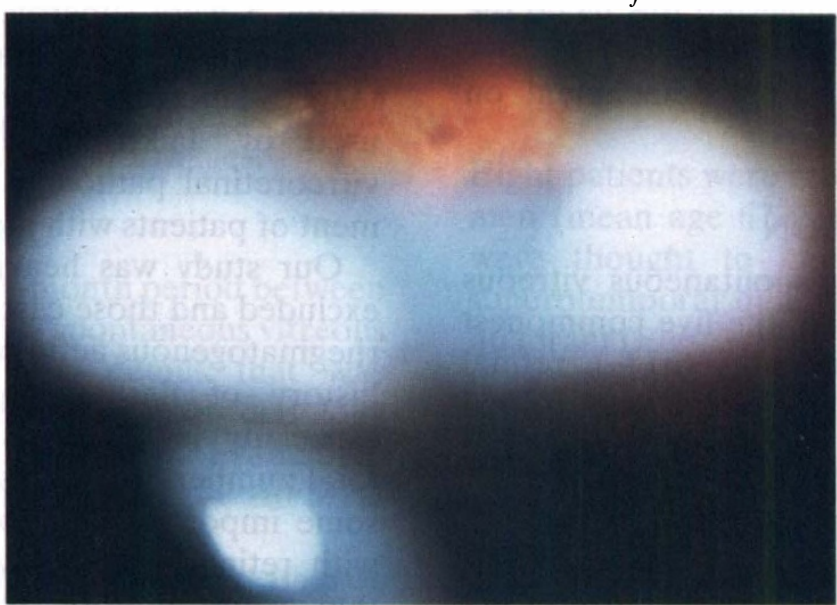

Fig. 7. Peripheral retinal tear from the study; the posterior margin of the tear is seen inferiorly with the edge of the operculum seen superior to this. There is haemorrhage at the apex of the operculum with fresh laser burns seen to one side. 


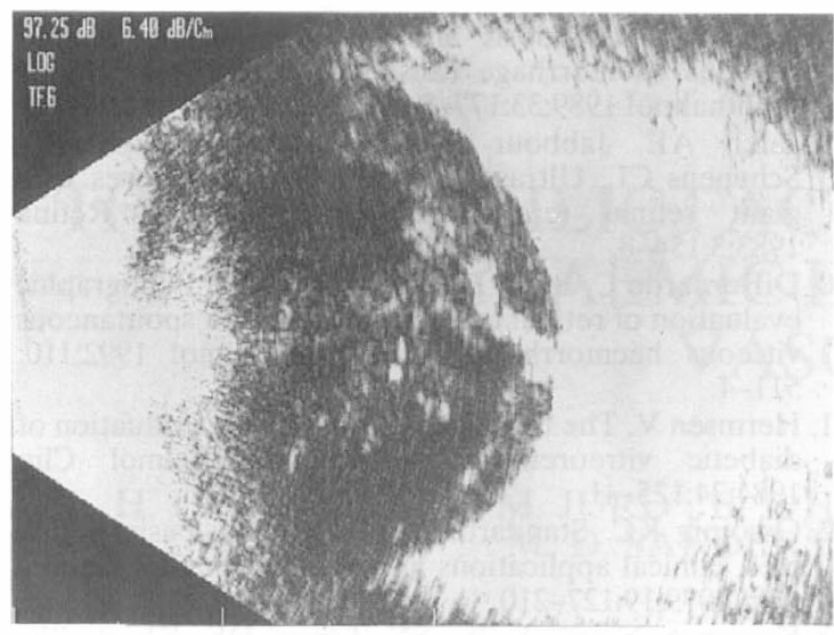

Fig. 8. Vitreous haemorrhage in a diabetic patient not in the study, shown on vertical section B-scan; the features of a neovascular frond are illustrated (see text). Acoustic enhancement is clearly seen along the area of the posterior vitreous attachment.

retinal tears also displayed a posterior vitreous detachment with the posterior vitreous face attached to the apex of the flap, while a dynamic scan revealed a hinge-like motion of the flap with a whip-like motion of the vitreous if the patient was asked to look to the right and then left quickly. These findings are similar to those described by others. ${ }^{10}$

Three previous studies ${ }^{5,8,9}$ incorporating ultrasonography in the diagnosis of spontaneous vitreous haemorrhages suggested that retinal tears needed to be large or giant in order to be detected. All three studies used an immersion scanning technique with the patient supine. We feel that one of the main reasons we were able to detect tears of a small to moderate size was that the patients were being scanned in the upright position, thereby utilising the full effect of gravity to pull the flap of the tear open, making it easier to detect. Furthermore, the advantage of the contact method is that the probe can be moved over the globe more easily and to a greater extent, allowing better access to scans of the periphery.

DiBernardo et al. ${ }^{10}$ described diagnosis of retinal tear in $17(40 \%)$ patients of 42 with spontaneous vitreous haemorrhage (diabetics excluded) in a retrospective study; the tears were found to be of small to moderate size. They used a contact scanning technique in a supine patient with the eye open, suggesting that scanning through the lids caused too much attenuation of the ultrasonic waves. In this study one retinal tear was missed and one neovascular frond mistaken for a tear. We scanned all patients in an upright position using the contact method through the closed lids. The latter did not prevent adequate analysis of the tears in our series.

The main differential diagnosis of a retinal tear in

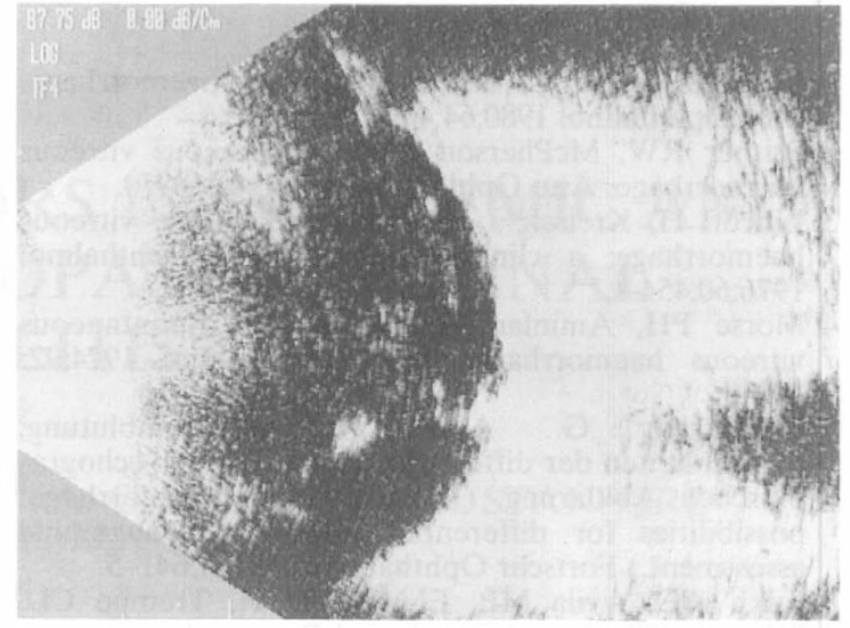

Fig. 9. Neovascular frond seen at the disc on a horizontal section B-scan in a diabetic (not in the study) presenting with spontaneous vitreous haemorrhage.

a spontaneous vitreous haemorrhage is a neovascular frond, as this structure also extends from the retina to the posterior vitreous face; however, they are usually well in the posterior pole (unlike most tears), show no discontinuity in the retinal echo, and there is usually acoustic enhancement where they attach to the posterior vitreous face (Figs. 8, 9).

\section{CONCLUSION}

The incidence of retinal tear in spontaneous vitreous haemorrhage in a non-diabetic is high. To help detect retinal tears using dynamic ultrasound scanning we suggest that the patient be examined in the upright position (in the first instance: see above) with the contact scan method; this can be done through the open or closed eye, though we found that since scanning through the lids was more comfortable for the patient a more thorough scanning protocol could be adhered to. However, in cases where the examiner is not sure about the nature of the structure being examined, scanning on sclera may reduce signal attenuation somewhat. A high-density real-time video display makes analysis of the kinetics of the vitreous, vitreoretinal interface and any structures arising from the retina much easier. Once a possible tear is diagnosed, frequent follow-up with repeat scans should be done until the fundus can be visualised and appropriate treatment given; if during follow-up scans a detachment is thought to be developing, repair can be offered utilising vitrectomy.

Our study suggests that the detection of small to moderate size retinal tears in spontaneous vitreous haemorrhages is possible using dynamic ultrasound scanning in the manner described.

Key words: Dynamic ultrasound B-scan, Retinal tear, Spontaneous vitreous haemorrhage. 


\section{REFERENCES}

1. Lean JS, Gregor Z. The acute vitreous haemorrhage. Br J Ophthalmol 1980;64:469-71.

2. Butner RW, McPherson AR. Spontaneous vitreous haemorrhage. Ann Ophthalmol 1982;14:268-70.

3. Lincoff $\mathrm{H}$, Kreissig J, Wolkstein M. Acute vitreous haemorrhage: a clinical report. $\mathrm{Br} \mathrm{J}$ Ophthalmol 1976;60:454-8.

4. Morse PH, Aminlari A, Scheie HG. Spontaneous vitreous haemorrhage. Arch Ophthalmol 1974;92: 297-8.

5. Hasenfratz G. Akute Glaskorpereinblutung: möglichkeiten der differentialdiagnostischen, echographischen Abklarung. (Acute vitreous haemorrhage: possibilities for differential diagnostic echographic assessment.) Fortschr Ophthalmol 1990;87:641-5.

6. Jalkh AE, Avila MP, El-Markabi H, Trempe CL, Schepens CL. Immersion A and B scan ultrasonography: its use in preoperative evaluation of diabetic vitreous haemorrhage. Arch Ophthalmol 1984; 102:686-90.

7. Green RL. The echographic evaluation of spontaneous vitreous haemorrhage. In: Ossoinig $\mathrm{KC}$, editor. Ophthalmic echography. Dordrecht, the Netherlands: W. Junk, 1984:233-8.

8. Katsumi O, Hirose T, Kruger-Leite E, Kozlowski I,
Tanino T. Diagnosis and management of massive vitreous haemorrhage caused by retinal tear. Jpn $\mathbf{J}$ Ophthalmol 1989;33:177-84.

9. Jalkh AE, Jabbour N, Avila MP, Trempe CL Schepens CL. Ultrasonographic findings in eyes with giant retinal tears and opaque media. Retina 1983;3:154-8.

10. DiBernardo C, Blodi B, Frazier Byrne S. Echographic evaluation of retinal tears in patients with spontaneous vitreous haemorrhage. Arch Ophthalmol 1992;110: $511-4$.

11. Hermsen V. The use of ultrasound in the evaluation of diabetic vitreoretinopathy. Int Ophthalmol Clin 1984;24:125-41.

12. Ossoinig KC. Standardised echography: basic principles, clinical applications and results. Int Ophthalmol Clin 1979;19:127-210.

13. Kumar A, Verma L, Jha SN, Tewari HK, Khosla PK. Ultrasonic errors in analysis of vitreous haemorrhage. Indian J Ophthalmol 1990;38:162-3.

14. Byer NE. Clinical study of retinal breaks. Trans Am Acad Ophthalmol Otolaryngol 1967;71:461-73.

15. Clemens S, Gerding H, Emmerich K-H. Dosierung der retinalen Kryotherapie mit hilfe der Echographie. (Use of ultrasonography to monitor retinal cryotherapy.) Klin Monatsbl Augenheilkd 1991;199:12-5. 